\title{
LA VILLE COMME TEXTE SÉMANTIQUE DE LA CITÉ ROYALE EN ASIE DU SUD (note critique)
}

\author{
GÉRARD TOFFIN
}

Une sémiologie des espaces urbains est-elle possible? Dans une étude pionnière, parue en 1967, en pleine marée structuraliste, Françoise Choay répondait à cette question par l'affirmative ${ }^{1}$. Malgré leur hétérogénéité, les villes, toutes les villes, seraient «saturées de significations » et travaillées d'images. Elles seraient fondées sur un système d'éléments signifiants apparentés au texte écrit et pourraient donc être appréhendées selon les mêmes méthodes que celles de la linguistique générale. Une telle sémiologie de la cité ne serait pas seulement possible mais souhaitable : les urbanistes y trouveront peut-être «le fil conducteur qui leur permettra de donner aux nouveaux ensembles urbains la richesse de significations dont ceux-ci sont aujourd'hui dépourvus » (p. 11). Quelques années plus tard, Roland Barthes explorait à son tour le champ sémantique du domaine bâti et réfléchissait à ses fondements linguistiques ${ }^{2}$. Sans chercher à présenter ses résultats sous une forme véritablement positive, entendons scientifique, il déchiffrait à l'aide d'un tel prisme la ville de Tôkyô - ville du «signe fort », en dépit de ses signifiés fuyants sinon flottants.

Cette façon d'envisager les problèmes d'urbanisme se rattache au grand tournant linguistique des années cinquante et soixante. Pendant une vingtaine d'années, on le sait, les questions relatives au langage envahirent le territoire des sciences humaines. Des concepts linguistiques, tirés de l'analyse phonologique et de la sémiologie, furent appliqués, sans beaucoup de discernement, à des domaines très divers : système de parenté, conte populaire, mythe, œuvre d'art, vie psychique, conduite individuelle, etc. Pour Lévi-Strauss et quelques autres, il s'agissait de tirer parti de la phonologie structurale pour fonder une analyse véritablement scientifique des faits sociaux et des diverses manifestations de l'esprit humain. Pour un petit groupe de philosophes, dont Derrida, le langage, toujours antérieur à la conscience, ouvrait un champ de recherche qui per-

* A propos de James S. Duncan, The City as text: the politics of landscape interpretation in the Kandyan kingdom, Cambridge, Cambridge Univ. Press, 1990, 229 + xIv p., cart., tabl., index, bibliogr. 


\section{TERRITOIRES URBAINS}

mettait de dépasser les vieilles oppositions jugées périmées entre la lettre et l'esprit, le réel et la fiction. De toutes parts, on se convertit au langage et l'on découvrit l'écriture derrière la parole. En France, ce «mirage linguistique», pour reprendre l'expression de Thomas Pavel ${ }^{3}$, a perdu aujourd'hui de son pouvoir d'attraction. La volonté de voir partout des signes et de réduire toutes les pratiques sociales à des questions langagières se révéla trop insistante pour ne pas être jugée rapidement excessive. De même, les espoirs entretenus par quelques-uns de fonder l'ensemble des sciences humaines sur l'analyse des systèmes de communication furent vite déçus. La linguistique perdit peu à peu son rang de science pilote et la sémiologie, une discipline aux frontières particulièrement élastiques, eut beaucoup de mal à atteindre sa majorité scientifique — du moins par rapport aux objectifs qu'elle s'était fixée.

Les questions de sémantique restent cependant très présentes en architecture et en urbanisme, surtout aux États-Unis. La dimension symbolique de la ville, qui relève indiscutablement d'une théorie générale des significations, fait ainsi depuis quelques années l'objet d'une attention redoublée de la part de chercheurs appartenant à la mouvance post-structuraliste. Les phénomènes de mode mis à part, cet aspect s'avère d'une importance toute particulière pour l'analyse des villes de type «exotique », villes préindustrielles, agraires, à charge symbolique puissante et à évolution historique lente. Ces espaces urbains «hyper-signifiants » ne sont-ils pas, comme le remarquait Choay, plus propices que d'autres à un traitement de type linguistique? Ne véhiculent-ils pas, comme la langue, des façons de penser, de classer, de grouper, bref tout un appareillage cohérent d'oppositions et d'homologies? Cet arrangement réglé d'éléments est du reste aussi inconscient aux citadins que les règles phonologiques et syntaxiques aux locuteurs d'une langue particulière.

Où en sommes-nous aujourd'hui? Que ces villes «traditionnelles », les seules à nous retenir ici, soient porteuses de significations riches et multiples, qu'elles participent d'un système de représentations mariant divers plans, social, religieux, politique, économique, grâce à un réseau serré de correspondances, c'est un point acquis. Que ces systèmes d'idées n'appartiennent pas seulement aux «ordres conçus» mais aussi aux " ordres vécus", selon les mots de Lévi-Strauss, fixant les actes quotidiens des individus dans leurs plus infimes détails, voilà aussi un fait fermement établi. Nous savons également que de telles structures symboliques permettent d'intégrer l'individu à un ordre moral englobant qui n'accorde guère de place à la personne au sens sociologique du terme et n'autorise que peu de distance entre le citadin et le cadre construit dans lequel il vit. Plusieurs interrogations malgré tout subsistent : comment ces dispositifs symboliques fonctionnent-ils concrètement? Est-il possible d'en faire une description qui ne soit ni verbeuse ni gratuite? Quel ordre établissent-ils entre les différents éléments ou plans qu'ils rassemblent ? Ces représentations sont-elles partagées par tous?

Le livre de James S. Duncan, The City as text, consacré aux anciennes cités royales de Sri Lanka, permet de faire le point sur ces questions et d'aller plus loin que les quelques généralités d'usage en la matière. Par-delà son intérêt régional (qui n'est pas mince) ${ }^{4}$, cet ouvrage, écrit par un géographe, présente en outre la particularité d'associer une approche sociologique du symbolique, cohérente quoique contestable, à une analyse sémiologique extrêmement fouillée. La dimension sémantique, langagière, polysémique de la ville, est ici replacée dans le cadre historique et politique de l'époque; elle est contextualisée. Bien qu'à peine ébauchée, cette synthèse entre récit textuel et histoire, narrativité et événement, est trop rare pour ne pas être saluée. C'est à cette tentative que je m'attacherai ici. Elle appelle toutefois le débat et pose de nombreux problèmes d'interprétation générale qui méritent d'être discutés. 


\section{La ville, miroir du cosmos}

Soit donc Kandy, centre politique et religieux de première importance dans l'histoire culturelle de Sri Lanka, dernière capitale cinghalaise, tombée entre les mains des Anglais en 1815. De quoi parle cette ville à la fin du XviII ${ }^{\mathrm{e}}$ siècle ? Que symbolise-t-elle? Pour Duncan, Kandy serait "informée » par deux modèles différents, deux conceptions opposées de la royauté. Le premier modèle, qualifié d' "Açokan », du nom du célèbre empereur indien converti sur le tard au bouddhisme, met l'accent sur les qualités morales du roi, sa piété, sa dévotion, sa droiture, sa mansuétude, la protection dont il entoure le bouddhisme et ses obligations (abondance, prospérité) vis-à-vis de ses sujets. Le souverain est vu à l'instar d'un bodhisattva, être surnaturel tout puissant, rayonnant, mais néanmoins attentif au sort des humains. Le second modèle, appelé «Çakran », fait du roi une réincarnation du dieu Çakra, le roi des dieux (= Indra); il le décrit régnant sur ses sujets comme Çakra règne dans l'empyrée sur les puissances divines. Le souverain est également imaginé sur le prototype du souverain universel cakravartin : il tourne la roue de la Loi pour maintenir l'ordre socio-cosmique du monde. Cette dimension, plus charismatique que la première, insiste sur les aspects sacrés et glorieux du roi. Des deux discours, le premier ressortit plutôt au bouddhisme, le second à l'hindouisme - les deux traditions religieuses constitutives de la civilisation cinghalaise. Mais ils $s$ 'interpénètrent étroitement et étaient mobilisés alternativement par le monarque afin d'affermir son pouvoir selon les nécessités du moment.

Ces deux modèles engendreraient chacun une forme particulière d'urbanisme. La conception royale «Açokan » favoriserait la production «d'un paysage urbain dominé par les structures religieuses et les travaux publics entrepris pour le bien-être de la population » (p. 6). La conception "Çakran», développée plus tardivement à Kandy, vers le $\mathrm{x}^{\mathrm{e}}$ siècle ap. J.-C., donnerait naissance à une cité cosmique modelée à l'image de celle du roi des dieux dans le ciel : elle augmenterait la distance entre le monarque et ses sujets. Les deux paradigmes de la royauté - qu'avait distingués en son temps l'anthropologue Obeyesekere $^{5}$ - sont commentés dans de nombreux textes religieux, le plus souvent pan-indiens, ainsi que dans une riche tradition orale qui définit les règles de bonne conduite du souverain. C'est sur ces textes (dont l'Arthaçâstra, connu à Sri Lanka de longue date) et ces traditions que l'urbanisme cinghalais est basé.

Comment ces systèmes de représentations sont-ils concrètement insérés et traduits dans le tissu urbain? Prenons l'exemple du modèle "Çakran ». Deux thèmes narratifs complémentaires peuvent être identifiés. Le premier, le plus important, assimile le paysage urbain à la demeure céleste des dieux. Il s'articule autour de trois signifiés : la cité idéale de Çakra, le mont Meru (axe cosmique de l'univers) et l'Océan primordial de lait d'où le monde a surgi. De nombreux éléments architecturaux renvoient à ces référents mythiques. C'est le cas en particulier du palais, cerné par une véritable forêt de symboles. Fondamentalement, la demeure royale est conçue comme la réplique de la montagne cosmique Meru au centre de l'univers. Le roi dans son palais apparaît comme le dieu Çakra au sommet du Meru, sa résidence, c'est-à-dire comme un dieu sur terre. En outre, le palais est censé reposer sur une tortue blanche, animal mythologique dans le monde indien s'il en est, avatar du dieu Viṣnu, soutien d'une autre montagne cosmique, le Mandara, que les Asura et les Deva utilisèrent à l'aube du monde pour barrater l'Océan de lait primitif dont sont sortis, entre autres, divers symboles de fécondité et insignes de royauté. La présence de cet Océan de lait, que la pensée mythologique situe au pied de la montagne cosmique, est d'ailleurs attestée métaphoriquement au sud du palais par un lac artificiel appelé Kiri Muhuda, aménagé en 1812 par Çri Vikrama. Les 


\section{TERRITOIRES URBAINS}

indices cosmogoniques dont Duncan fait la liste (tours octogonales, piliers à quatre sections, pierres de lune, éléphants sculptés, Ficus religiosa, etc.) sont légion. On a là une analyse, fine et exhaustive, qui mériterait d'être transposée à d'autres ensembles royaux d'Asie du Sud. Quant au second modèle "Açokan», il assimile le paysage urbain de Kandy aux premières capitales royales de Sri Lanka (Anuradhapura, Polonnaruva), gouvernées par des rois répondant à la figure prototypique du cakravartin et construites elles-mêmes à l'image des cités célestes. Trois préoccupations s'y font sentir : le contrôle sur l'univers à travers le jeu des directions de l'espace, la reproduction en miniature d'éléments cosmiques, l'imitation des cités idéales des rois-héros cinghalais dont les hauts faits sont pieusement conservés par la mémoire collective.

A l'instar de la ville occidentale qui était vue au Moyen Age comme une Jérusalem céleste, la ville royale indienne et sri-lankaise (rajadhânî ou pura) est donc conçue à l'image de cités divines; elle correspond à un archétype. D'où sa dimension cosmique: l'espace urbain matérialise la topographie du cosmos dans ses moindres détails, il est le miroir de l'univers tout entier. Selon un modèle indien courant, Kandy était ainsi divisée en quatre secteurs, correspondant chacun à une direction de l'espace. Chaque partie était associée à une fête majeure, à un temple, à une porte, et même à un ferry qui permettait aux gens de l'extérieur d'accéder à la ville. Sous le règne du dernier roi de Kandy, 'Sri Vikrama, un grand bâtisseur, les quatre secteurs de la cité furent même subdivisés en seize carrés, peut-être pour s'approcher des indications de l'Arthaçâstra qui précise qu'une capitale idéale doit être basée sur un damier de quatre fois quatre cases ${ }^{6}$. A quoi répondait ce souci de reproduire le macrocosme au niveau du microcosme, de se conformer aux prescriptions des textes canoniques? Il s'agissait essentiellement de garantir à la ville royale une dignité religieuse qui puisse rejaillir à la fois sur son souverain et ses sujets. Le but était d'accorder la terre au ciel, d'effacer toute séparation entre humains et divins. Habiter un tel territoire, c'était du même coup participer à certaines correspondances cosmiques, vivre en harmonie avec l'univers dans un monde plein. Cette condensation de symboles sous l'égide des dieux donne à Kandy, comme à toutes les capitales " traditionnelles» d'Asie du Sud, une dimension nettement allégorique.

Mais il y a davantage. Si la cité royale a une résonance cosmique patente, elle incorpore également dans ses murs les éléments du royaume. Ce dernier, en effet, comprenait lui aussi idéalement quatre parties. De sorte qu'en contrôlant les quatre portes de la cité, le roi exerçait symboliquement son pouvoir sur la totalité de son royaume. A quoi il faut ajouter que le roi Çri Vikrama, dont il a déjà été question, divisa Kandy en 21 secteurs correspondant au nombre d'unités administratives dont le royaume était composé. Les gouverneurs de ces provinces vivaient apparemment chacun dans un des 21 quartiers de la ville.

En synthétisant ces données, Duncan verse une pièce supplémentaire à un dossier déjà très fourni. De nombreuses villes asiatiques, bouddhistes et/ou hindoues, se conforment à un tel modèle. De l'Asie du Sud à l'Asie orientale en passant par l'Asie du SudEst et le monde indonésien, la figure mythique du mont Meru, axe médiateur entre Terre et Ciel, est devenue un symbole essentiel de l'espace du temple et du palais, un paradigme de la royauté. En même temps, les murs entourant la plupart de ces cités figurent les limites du monde et la symbolique des quatre points cardinaux fixe, comme P. Wheatley l'a montré pour la Chine ${ }^{7}$, toute une topographie religieuse qui imprègne les représentations des citadins; l'espace urbain est en quelque sorte accordé aux forces transcendantes du cosmos. Dans les villes indiennes, ces notions de centre, de quartiers et de périphérie sont parfois explicitement utilisées par les religieux ou les peintres pour représenter la cité sous la forme d'une figure géométrique ${ }^{8}$. La ville devient alors un mandala, un cosmogramme, expression de l'unité profonde de l'univers.

Ouvrons ici une brève parenthèse à propos du palais, point focal de la ville (même 
s'il ne correspond pas toujours à son centre géométrique) et instance dirigeante de la société urbaine. Venant après celle de H. L. Seneviratne", la description de Duncan confirme deux traits caractéristiques du palais royal en Asie du Sud. La résidence du roi tout d'abord est construite à l'image de la cité (ou vice versa) - c'est une ville intérieure - , elle constitue à elle seule un concentré de l'univers, elle se suffit symboliquement à ellemême. Le palais, en second lieu, est associé à un temple. Qu'il soit situé dans le voisinage ou qu'il fasse partie intégrante de la demeure royale, ce sanctuaire légitime le souverain. A Kandy, il s'agit du Daladâ Mâligâva, le reliquaire de la dent du Bouddha, désigné par le même mot, mâligâva, que le palais (p. 109). Entre le sacré et le pouvoir les liens apparaissent profonds.

Mais revenons au symbolisme cosmique. Comment fonctionne cette sémantique de la ville? Il convient de remarquer au préalable que le processus de symbolisation ne s'exprime pas seulement sur un registre architectural, iconique, mais aussi sur le plan rituel. Considérons l'Asala Perahära, l'une des fêtes les plus importantes de Kandy, qui commémore à la fois le jour de la conception du Buddha Çâkyamuni et celui de son départ de la maison familiale. Cette célébration se tient tous les ans en juillet-août, lors de la pleine lune du mois Asala. Elle semble avoir deux objectifs étroitement liés: assurer la prospérité du royaume en maintenant un contact entre Terre et Ciel, et réaffirmer le pouvoir du souverain sur ses sujets. Toute la population y participait, y compris les représentants des différentes provinces et les paysans de l'arrière-pays. Le rituel commençait par l'érection d'un mât, kapa, symbole de celui d'Indra (Çakra), indrakîla, et de l'arbre mythique kapruka, qui sortit de l'Océan de lait au moment du grand barattage. Ce mât représente l'axis mundi, trait d'union entre les Trois Mondes. Il était placé au centre d'un diagramme, astamagala, délimité par les huit points cardinaux et arrosé d'eau. Puis la sainte relique du Buddha, habituellement conservée dans le Daladâ Mâligâva, à côté du palais, était portée en procession dans la ville, suivie par le roi et les dignitaires du royaume montés sur des chevaux et des éléphants. Ce rite, qui fut transformé selon D. Winslow " en fête bouddhiste nationale par le roi Çri Râjasimha (17471782), contient de nombreuses allusions mythologiques et des éléments de la tradition littéraire indienne. Elle met en scène, comme l'architecture monumentale, les conceptions royales "Çakran" et "Açokan", réactualisant tous les ans les fondements religieux de l'urbanisme cinghalais auprès de la population.

Duncan fait par ailleurs ressortir avec raison le rôle des systèmes de nomination dans ce langage symbolique. C'est un point sur lequel on n'insiste généralement pas assez. Le fait d'appeler le lac qui borde sa résidence "Océan de lait», Kiri Muhuda, n'est pas indifférent. Il s'agissait pour le roi et ses prêtres de mêler l'espace mythologique à l'espace urbain et de placer plus fermement la capitale royale au centre de l'univers. En donnant aux monuments des noms prestigieux tirés d'un corpus littéraire, les dirigeants opéraient une analogie: ils renvoyaient l'objet considéré à un système de valeurs sousjacent, historique ou idéal. Comme l'écrit Anne Cauquelin dans son beau livre L'invention $d u$ paysage, "Le nom fait valoir ce qui dans un lieu est plus lieu que le lieu même " $"$. C'est sous la protection des noms que l'on se place. A cet égard, les paysages urbains d'Asie du Sud peuvent être considérés comme une suite de citations inscrivant l'ordre physique, écologique, de la ville à l'intérieur d'un système de référence.

\section{Une rhétorique du paysage urbain?}

Nous voici au cœur du livre: la textualité présumée de la ville. Cette thèse, nourrie de Barthes, de Certeau et Geertz, se fonde sur deux types d'arguments. Duncan souligne 


\section{TERRITOIRES URBAINS}

d'abord que Kandy (comme toutes les cités royales indianisées) est une société hautement «textualisée » (pp. 22-23) : même s'il n'est pas compris par tous, l'écrit y occupe un statut élevé; la vérité, estime-t-on, sort des livres. Le code urbain, on l'a vu, y est constellé de signes, d'indices, de traces qui renvoient à des textes religieux, hindous ou bouddhistes. Or ces textes, qui appartiennent à la tradition indienne classique, contiennent des indications sur la façon dont une ville doit être construite, sur ses dimensions, ses orientations, l'emplacement de ses divers monuments et de ses groupes sociaux. Mon expérience de la vallée de Kathmandou, autre riche civilisation urbaine d'Asie du Sud, corrobore ici en tout point la thèse de l'auteur. Dans cette vallée de l'Himalaya, les modèles textuels restent très présents; il y est fait fréquemment référence quand on construit un monument ou qu'on accomplit un rituel. Il n'est certes pas possible de soutenir que les villes du monde indien, au sens large, sont nées de paradigmes textuels, mais elles sont indiscutablement basées sur une interpénétration poussée entre le physique et l'écrit.

Second argument, lié au premier: la ville est une combinaison de signes, un idéogramme. Or, le langage de la ville, comme celui des mots, procède par métaphores, imitations, c'est un tissu d'allusions. Il met en œuvre les traits caractéristiques de tout processus symbolique, alternant sens directs, sans détours, et significations indirectes, transposées. Ce point, lui aussi, paraît difficilement réfutable : les signes dont les villes asiatiques - toute ville - sont formées, sont toujours marqués, positifs ou négatifs, attirants ou repoussants, mais jamais neutres. Duncan propose de les analyser au moyen de quelques tropes linguistiques: la synecdocque, qui consiste à prendre la partie pour le tout ou inversement, la métonymie, dans laquelle un mot, une image, tiennent lieu de quelque chose d'autre avec lequel ils sont en relation de contiguité, l'allégorie et la récurrence. Ce sont ces tropes qui donneraient à la ville un statut textuel. Le paysage urbain, en définitive, fonctionnerait comme un système d'écriture parce qu'il est porteur de sens et qu'il repose sur un ensemble articulé de concepts.

Est-ce suffisant pour parler de texte? S'il est vrai que des domaines non proprement linguistiques, comme le mythe ou la parenté, constituent des systèmes symboliques et peuvent, à ce titre, être considérés comme des langages particuliers, est-on pour autant en droit de les qualifier d'écritures? Ces "textes» inscrits dans la pierre peuvent-ils valablement être comparés à l'écriture sur le papier? Pour dépasser la simple métaphore, parlante mais contestable, il faudrait en réalité pouvoir mener à bien trois opérations: dissocier le «texte» urbain en unités discontinues, distribuer ces dernières en classes formelles et, enfin, mettre en évidence les règles de combinaison et de transformation de ces unités. Duncan tente de faire ce travail. Grâce à ses deux modèles royaux, "Açokan» et "Çakran », il réussit à isoler deux univers de référence: "le monde des héros », et "le monde des cakravartin », eux-mêmes subdivisés, comme on l'a dit, en thèmes narratifs. Il ne lui reste plus alors qu'à classer les signes architecturaux de la ville dans l'un ou l'autre de ces modèles ou thèmes de référence. Le résultat est une série de tableaux d'une extrême précision qui, surtout en ce qui concerne les monuments centraux de la ville, dressent une liste des synecdocques selon les trois registres distingués: iconique, onomosmatique ( = métonymique) et rituel. Ainsi se trouve esquissée une grammaire du symbole qui éclaire la poétique de la cité.

Beaucoup ne seront pas convaincus par cette algèbre linguistique. D'autres admettront, comme moi, qu'à défaut d'identité complète, l'interprétation du symbolisme urbain mérite d'être rapprochée de l'exégèse textuelle. Il y a indiscutablement analogie entre le système de communication linguistique et le langage des signes urbains. Bien qu'appartenant à deux ordres de réalité différents, ces deux systèmes de significations sont des phénomènes du même type, au moins quant à la forme. Faute de compétences 
particulières, je ne me hasarderai cependant pas plus avant dans un domaine où chaque linguiste, ou presque, défend ses propres définitions. Deux remarques suffiront ici. Premièrement, en dépit de leurs valeurs théoriques et méthodologiques, ces notions ne nous apprennent rien sur le symbolisme cosmique de la ville asiatique que nous ne sachions déjà. Leur valeur heuristique apparaît singulièrement limitée. Deuxième remarque: Duncan propose dans son schéma une correspondance terme à terme du symbolisme. Il assigne à chaque signifiant un ou deux thèmes narratifs particuliers. Mais s'il est une chose que les études de sémantique nous ont apprise, c'est que les rapports entre le signe et le référent sont autrement plus labiles, voire indéterminés. Il est rare qu'un signifié corresponde à un signifiant unique. Ce dernier n'excède-t-il pas toujours le concept qu'il contient? The City as text accorde en vérité une importance exagérée au lexique, lequel n'est pas le meilleur outil, loin de là, pour étudier un système de signes. Il n'est plus possible, aujourd'hui, de chercher un sens unique, scientifiquement défini, à tel ou tel écrit. Tout texte est par nature polysémique et admet plusieurs lectures. Il serait sans doute excessif d'affirmer qu'il y a autant de villes que de points de vue, mais force est de reconnaître que la multiplicité des interprétations possibles relativise sérieusement la transposition mécanique qui nous est présentée ici.

On regrettera du reste que cette analyse sémiologique laisse de côté le domaine bâti, habité. Cet espace construit possède pourtant, lui aussi, un vocabulaire, une syntaxe faite de répétitions, de symétries et d'oppositions. Son champ sémantique compose une part essentielle de la symbolique urbaine, comme il l'a été montré pour d'autres villes d'Asie du Sud ${ }^{12}$. En centrant son étude sur les seuls monuments, Duncan a délibérément privilégié le plan paradigmatique de la ville auquel s'attache la mémoire collective du groupe et évacué du même coup le plan syntagmatique qui touche aux faits de proximité, de contiguïté (îlot, rue, façade). Sa ville, d'encre et de papier, en raison des contraintes de l'écriture, n'opère qu'au niveau du monumental, non du quotidien vécu et du cadre de vie.

Une autre question reste ouverte : quel impact les idées religieuses ont-elles eu sur la morphologie de la ville? Jetons un coup d'oeil sur le plan général de Kandy. La cité est divisée en quatre parties par deux rues principales qui se croisent à angle droit. Ces deux axes donnent sa structure de base au tissu urbain. Celui qui traverse la ville d'est en ouest partage Kandy en deux unités administratives dirigées chacune par un "gouverneur ", adikâr, du roi. Significativement, ces deux hauts fonctionnaires administraient aussi les deux moitiés correspondantes du royaume (p. 92). L'autre axe, nord-sud, divise la cité en deux rectangles: occidental et oriental. Le premier était réservé aux nobles, aux gens du commun et au commerce. Dans le second, d'un statut plus élevé, s'élevaient le temple et le palais. Ce quadrillage de la cité, accordé aux directions cardinales, relève d'emblée d'une vision cosmogonique. La quadripartition de l'espace urbain, on l'a vu, est attestée dans les textes sanskrits et découle d'un symbolisme général en Asie. Quant à la division en deux rectangles, est et ouest, elle renvoie, elle aussi, à une forme idéale de la ville céleste. On sait également que la pensée indienne valorise davantage le nord et l'est, directions des dieux et des sommets, que le sud et l'ouest, liés aux morts, aux démons et entachés d'impureté. La place du palais et du temple n'est donc pas déterminée au hasard, elle correspond à une sémantique des points cardinaux.

S'agit-il d'un urbanisme volontaire? Kandy était-elle totalement calquée sur les paradigmes célestes décrits dans les textes locaux ? Quelle est la part du modèle et de la réalité ? Les éléments manquent pour répondre de manière définitive à ces questions. Il faudrait en savoir plus sur l'histoire urbaine, les étapes de la croissance, les usages du sol, les logiques sociales en présence. On ne peut cependant s'empêcher de s'interroger 
sur le rôle des préceptes textuels ${ }^{13}$. Il est rare, en Asie comme ailleurs, qu'une ville soit modelée dès le départ à l'image d'une cité idéale. Dans la plupart des cas asiatiques que nous connaissons, la morphologie urbaine résulte plutôt de l'addition de logiques familiales et de quelques initiatives individuelles. Les facteurs sociaux et les structures familiales notamment jouent un rôle considérable dans la dynamique du tissu urbain ${ }^{14}$. Les paradigmes religieux indiens n'ont-ils pas été appliqués après coup (à Kandy: tardivement, d'après ce que l'on sait) sur une ville déjà plus ou moins existante ? On peut raisonnablement le penser. L'assise géométrique de Kandy, avec son centre et sa structure bipolaire, est au demeurant trop universelle pour qu'on puisse y voir l'application immédiate de schémas indiens scripturaires. Toute ville s'articule autour des axes de circulation qui la traversent et il a été montré que les plans géométriques supportent de par le monde des intentions et des mécanismes fort variables ${ }^{15}$. Qu'on ne se méprenne pas : je ne cherche pas à nier l'impact des idées religieuses. Leur rôle est indéniable, ne seraitce que dans les efforts déployés par certains rois pour élever le statut religieux de leur capitale. Il me semble seulement que cette image, par trop idéaliste, d'une ville entièrement «cosmicisée », toute vibrante d'harmonies célestes, ne correspond pas totalement à la réalité, et que, sous cet habillage religieux, se cache un autre urbanisme, plus secret : un urbanisme sans urbaniste, fait d'éléments fractionnés, de pièces juxtaposées - ce qui ne veut pas dire sans structure - dont le langage nous reste pour l'instant inconnu. La ville n'est jamais le simple fac-similé d'un canon céleste. Comment ne pas tenir compte des décalages entre image idéale de la cité et traces de constructions préexistantes? Là aussi, plusieurs lectures, dont il serait intéressant d'étudier les relations, s'avèrent possibles. Comme nos maîtres nous l'ont appris, la réalité de la représentation n'est pas toujours la représentation de la réalité, même si elle concourt à la constituer.

\section{Politique et urbanisme}

Duncan, reconnaissons-le, ne s'en tient pas à la symbolique de la ville. Il cherche également à mettre en évidence le rôle de ces représentations dans les pratiques sociales et politiques. La ville royale cinghalaise ne serait pas uniquement structurée - par des textes - elle serait structurante. C'est par son truchement que s'affirmerait en particulier l'autorité royale et que se reproduiraient les relations de pouvoir. En cas de crise ouverte, c'est autour de ses dispositifs métaphoriques que s'affronteraient les diverses factions du royaume. Le paysage urbain, autrement dit, serait un instrument politique, un outil idéologique. Le roi utiliserait les thèmes narratifs gravés dans les pierres de sa capitale pour légitimer sa couronne. Il ne s'agirait pas seulement pour lui d'impressionner ses sujets par la beauté de monuments remarquables, mais aussi d'user du parallèle entre cité royale et cité divine pour renforcer la dimension religieuse exemplaire qui est la sienne. En manipulant les symboles divins et les puissances surnaturelles, il sanctifierait, de manière intéressée, son pouvoir sur les masses. Citons l'auteur : «Il y a de bonnes raisons de penser que les "textes paysagés" étaient lus selon une perspective politique hégémonique. Le codage des architectures urbaines d'après les textes religieux et politiques était, en définitive, un procédé courant en Asie du Sud, du Sud-Est et en Extrême-Orient. Il est douteux que le souverain ait persisté dans cette voie coûteuse s'il n'en tirait pas quelques avantages » (p. 155). On serait d'autant plus en droit de parler d'idéologie que le paysage architecturé est ressenti par la population comme naturel, donné comme préétabli, alors qu'en fait, tout en lui est construit.

C'est dans ce contexte qu'il faut envisager l'influence croissante au fil des années du modèle charismatique à Kandy. Duncan, qui n'explique pas réellement cette évolution, 
se place dans la dernière phase du royaume, de la fin du XVIII ${ }^{e}$ siècle à l'aube du XIX ${ }^{e}$ siècle. La dynastie étrangère (sud-indienne) Nâyakkar détient alors le pouvoir. Dans un climat politique tendu (les Anglais exercent une forte pression dans l'île), Çri Vikrama, le dernier souverain de Kandy, promeut le modèle "Çakran " dans sa capitale au-delà de tout ce qui avait été fait jusqu'alors. Il se lance dans une politique de grands travaux urbains pour rehausser le statut "divin » de Kandy (et de sa royale personne). Mais les confiscations de terre et l'augmentation des obligations de travail gratuit (râjakariya) qu'il est contraint d'imposer à cet effet aux paysans lui aliènent une grande partie de la population. L'élite bouddhiste, en conflit ouvert avec la famille royale depuis le début du Xvirr ${ }^{e}$ siècle, accuse le roi de confisquer le pouvoir à son seul profit, sans se préoccuper du bien-être de ses sujets, et de céder à la folie des grandeurs. S'appuyant sur le modèle "Açokan" elle cherche par tous les moyens à déstabiliser la maison royale Nâyakkar. Après maints événements sanglants, ces notables pactisent en secret avec les Anglais en 1815 et leur livrent la ville. Ils seront vite déçus car, contrairement à ce qu'ils avaient espéré, les Anglais ne leur cédèrent pas le trône. Kandy perdit son autonomie et se vit intégrée à l'Empire colonial britannique.

L'utilisation de modèles religieux à des fins politiques apparaît aussi clairement lors des fêtes urbaines. Ces savantes mises en scène liturgiques n'étaient pas entièrement gratuites : elles rechargeaient, à périodes rapprochées, l'aura sacrée dont le souverain était nimbé. Elles rappelaient qu'un roi est au pouvoir et qu'il faut lui obéir parce qu'il est le grand organisateur de la religion, le garant de l'ordre cosmique et l'épicentre de la cité. Les processions, surtout, structures maîtresses de ces fastes royaux, favorisaient l'unanimisme: chacun y participait selon une place définie par son rang et sa dignité. On devine les implications: les cortèges composaient un répertoire hiérarchique de la cité, rendant immédiatement compréhensibles à la masse des spectateurs l'ordre social ainsi que les idées forces du système des castes. Ils persuadaient les participants que la ville, lieu d'harmonie physique et sociale, est un édifice commun avec à sa tête un roi. Leur enjeu était d'une telle portée qu'ils faisaient l'objet de querelles de préséance acharnées.

L'efficacité politique des idées inscrites dans l'architecture et mises en œuvre dans les liturgies urbaines prête cependant à discussion. Peut-on poser un lien de causalité aussi net entre langage symbolique et pouvoir effectif de la dynastie royale? L'approche de Duncan ne pêche-t-elle pas par finalisme? N'est-elle pas tributaire d'un sociologisme passé de mode? Le religieux, en Asie du Sud, se réduit-il, en dernière instance, à une affaire de pouvoir? Le souverain usait-il des représentations hindoues et bouddhistes à sa guise? Cette grappe de questions soulève des problèmes fondamentaux qu'il serait prétentieux de vouloir résoudre ici. Contentons-nous de quelques observations, particulières et générales, qui nuancent la thèse de Duncan. On observera d'abord que les rapports entre religieux et politique se posent en des termes différents en Occident et dans le monde indien. Dans notre univers christianisé, le spirituel et le temporel ont chacun leur logique et leurs valeurs propres. En dépit de certaines interférences, ils existent indépendamment et constituent des sphères autonomes. En Inde, à Ceylan, au Népal en revanche, il y a compénétration entre les deux ordres. Il n'y a pas deux règles de vie, l'une pour ce monde-ci, l'autre pour l'au-delà ; l'une et l'autre sont solidaires et forment un tout indémêlable. Il est vrai qu'à la différence du bouddhisme, la pensée hindoue établit une distinction entre pouvoir, kșatra, et religion, dharma. Il est vrai aussi que cette pensée n'admet pas de culte autour de la personne royale comme ce fut le cas dans certains pays d'Asie du Sud-Est indianisée (Cambodge, Indonésie). Mais le pouvoir reste dans l'hindouisme fortement sacralisé. Le roi et les dieux se trouvent situés dans un rapport d'homologie: les souverains sont dans leur palais comme les dieux dans leur temple, et vice-versa. De plus, hindouisme et bouddhisme définissent les 


\section{TERRITOIRES URBAINS}

relations du sacré et du temporel en des termes hiérarchiques. Ils accordent tous deux au spirituel une supériorité sur le séculier : rien n'est plus haut à leurs yeux que le brâhmane, le renonçant, le moine bhikșu ; ces figures emblématiques incarnent les valeurs ultimes de la société. Dans le monde hindou en vérité, l'impérium ne peut se maintenir sans le sacerdoce, seul garant de sa légitimité. Le roi se détermine par rapport à un ordre divin qui lui est transcendant: il sert les dieux, il se montre attentif à leurs désirs et compréhensif à l'égard de leurs exigences. En bref, dans ce type de culture, la religion est constitutive de la société. Les représentations symboliques n'affectent pas seulement le fonctionnement de la pensée, mais l'ensemble de la morphologie sociale et du système économique. Elles permettent aux individus de définir leur place dans le monde; plus que tout autre facteur, elles donnent un sens à leur vie.

C'est la raison pour laquelle la construction de monuments ne peut être imputée à de seuls motifs politiques. En finançant des bâtiments fastucux, les rois cherchaient aussi bien à manifester leur piété, leur dévotion; ils marquaient leur soumission à un ordre surnaturel dont ils admettaient volontiers qu'il les dépassait. Ils agissaient autant par nécessité religieuse que par calcul intéressé. De même, les liturgies urbaines ne peuvent être expliquées uniquement par des considérations politiques. Ces cérémonies étaient avant tout des rituels de régénération et de purification qui mettaient en branle un cortège d'idées religieuses, aussi présentes à l'esprit des participants que la puissance du roi. Partout dans le monde indianisé (et au-delà sans doute), les rituels urbains produisent de l'ordre plutôt que du désordre. Ils renforcent partout la hiérarchie sociale; ils canalisent les conflits, contrôlent les forces entropiques et créent des consensus. Ils n'en n'ont pas moins, pour les participants, une efficacité proprement symbolique, indispensable à la survie du groupe: faire tomber la pluie, assurer la récolte du riz, chasser l'ennemi de la ville, flatter et nourrir les dieux, etc.

La fonction royale repose donc sur des notions complexes, parfois contradictoires, toujours ambiguës, qu'il n'est pas facile de cerner. Comme l'avait remarqué Georges Bataille en son temps ${ }^{16}$, l'activité d'un roi, quel qu'il soit, se situe à l'intersection de deux logiques: le «travail», l' «utilité » d'une part, la «gloire», le «jeu», ce qu'il appelait le «sacré », d'autre part. D'un côté, l'efficience politique et la reproduction de l'ordre établi; de l'autre, le système symbolique dont le souverain n'est qu'un pion. Pour exister et durer, le roi est tenu d'alterner jeu et calcul : il se doit d'exprimer sa grandeur parce qu'il est souverain, mais il ne devient roi qu'en manifestant sa majesté. Il lui faut concilier ces deux aspects apparemment opposés. Et l'on s'exposera à ne rien comprendre à l'exercice de son pouvoir si l'on ne considère pas ensemble les deux versants. Il n'est, dès lors, guère possible de réduire l'architecture monumentale de Kandy à un quelconque sens univoque. La vérité d'un texte, si tant est qu'il en possède une, repose sur l'intention de l'auteur. Or, dans ce cas précis, il n'existe pas une seule motivation, mais plusieurs, juxtaposées ou superposées. Plutôt que de signe unique, mieux vaut parler d'un faisceau de significations concomitantes.

\section{Le pouvoir des symboles}

Qu'en est-il à présent de la réception de cette écriture gravée dans les pierres et inscrite dans les séquences rituelles? Était-elle lisible par tout le monde? Sans démontrer véritablement sa thèse, Duncan répond par l'affirmative (p. 152). C'est là un jugement lourd à porter. Que les Cinghalais de l'époque aient tous eu des notions de bouddhisme et d'hindouisme est une chose, qu'ils aient été à même de décrypter entièrement le symbolisme des monuments et des fêtes en est une autre. Les individus participant d'une 
même culture peuvent partager les mêmes symboles sans partager pour autant le contenu de ces symboles. Pour bien faire, il conviendrait de distinguer plusieurs niveaux de compréhension, donc de significations, dans les "textes" qui entouraient les habitants de Kandy. Certains éléments procédaient sans conteste d'un legs culturel commun, d'une mémoire collective remise en scène à chaque génération, et pouvaient, à ce titre, être le lot de tous. Mais il était des détails architecturaux et rituels dont le sens exact ne devait être connu que de quelques-uns, les prêtres par exemple, et qui nécessitaient un savoir particulier. Il est douteux que tous les sujets du royaume fussent à même de déchiffrer l'iconographie et l'architecture avec le même luxe de détails et de précision. Ils devaient, en réalité, en penser beaucoup moins que Duncan, qui s'aide de beaucoup de textes dont les quatre cinquièmes de la population cinghalaise n'avaient jamais lu une seule ligne pour reconstruire son système de significations. Aux yeux de la grande masse des citadins, le paysage urbain de Kandy apparaissait davantage comme un décor, un théâtre, un espace chargé de polarités religieuses, que comme un texte. La perception devait être globale, non analytique, à l'exception d'éléments particulièrement importants lors des fêtes ou réglant la circulation des personnes dans la cité. Le contrat de lecture, allégorique et métaphorique, qui nous est présenté s'avère par conséquent trop savant pour emporter la conviction. Comme Paul Veyne l'a rappelé récemment, le symbolisme présent dans une œuvre d'art n'est jamais efficace qu'à $10 \%$ de sa charge symbolique réelle ${ }^{17}$. Qui, parmi les habitants de Kathmandou au Népal, voit les sculptures érotiques qui ornent, haut placées et peu visibles, les consoles des temples? Qui est capable d'en expliquer la signification? Il faut distinguer vision et information. Les rituels, comme les espaces architecturaux, expriment par leur existence même plus qu'ils n'informent par leur contenu descriptif et discursif. P. Veyne écrit justement à ce sujet que ce type de monument parle avant tout «pour exprimer la puissance qui les a fait sortir de terre ${ }^{18}$. A Kandy comme à Kathmandou, les édifices, auxquels la population ne portait probablement qu'une attention distraite, n'exprimaient-ils pas surtout la supériorité des dieux sur les hommes et la puissance de la fonction royale?

Il est une autre critique, tout aussi fondamentale, que l'on peut opposer à la présumée fonction politique du symbole. A faire valoir de telles explications, ne risque-t-on pas de détourner les représentations religieuses et les rites de leur signification réelle? Un indianiste, F. Staal, grand spécialiste des rituels védiques, a récemment reproché aux anthropologues d'interpréter les rites par leurs seuls usages idéologiques ${ }^{19}$. S'en prenant en particulier à Clifford Geertz, auteur d'une importante étude sur les cérémonies royales de l'ancien royaume de Bali ${ }^{20}$, il accuse: "Le livre de Geertz traite de la politique, mais cela ne veut pas dire que les rituels balinais aient quelque chose à voir avec les relations de pouvoir " (p. 204). Pour Staal, il faut considérer les rites en soi, n'en rendre compte qu'en des termes tirés du système religieux local. En somme, si je comprends bien cet auteur, le symbole ne peut s'expliquer que par le symbole. Cette position anti-fonctionnaliste extrême est également défendue par certains anthropologues africanistes qui estiment que l'activité rituelle a sa propre logique, étrangère à la praxis politique. Les diverses opérations symboliques " produisant » la personne royale relèveraient d'un principe de répétition. Le rite ne viserait pas à diffuser un message idéologique, il répondrait à une logique obsessionnelle ${ }^{21}$ : a-t-il été effectué ? au bon endroit? à la bonne place?, etc. «La question de son sens serait extérieure à son effectuation $"{ }^{22}$. Prises en tant que telles, indépendamment des leçons qu'on peut en tirer à l'usage des peuples, ces opérations religieuses n'auraient d'autres effets que de conférer au souverain une existence cérémonielle échappant, autant que faire se peut, aux aléas du temps. Toutes ces remarques sont justes à leur manière. Elles ne nous apprennent cependant rien sur le pouvoir des symboles - d'emblée plus politiques, il est vrai, dans 


\section{TERRITOIRES URBAINS}

les civilisations urbaines que dans les espaces sociaux restreints d'Afrique noire. On peut du reste se demander si une culture donne spontanément à l'observateur étranger toutes les catégories d'analyse nécessaires à son interprétation. Faut-il se contenter d'une grammaire symbolique élaborée à partir de dires ou de textes locaux ? On peut en douter. Un bon chercheur en sciences humaines doit avoir deux oreilles: une pour écouter ce que le texte ou l'informateur lui dit, l'autre pour deviner ce que ce texte ou cet informateur ne veut pas lui dire.

On touche ici à la limite de ces critiques. A vouloir éliminer le sujet des représentations religieuses, ne risque-t-on pas de ne laisser au regard que des machines symboliques gratuites fonctionnant toutes seules? A déchiffrer les symboles en des termes purement religieux, ne risque-t-on pas de découper la réalité en une poussière de langages autonomes, tous équivalents? A refuser de se poser le pourquoi et le comment des choses, ne se condamne-t-on pas à tout diluer dans une structure aplatie, totalement isomorphe, où chaque élément réagirait sur chaque autre et réciproquement, sans relations hiérarchiques? C'est l'erreur d'un certain structuralisme d'avoir réduit l'étude des rites à l'étude du fonctionnement d'un système symbolique, reflet supposé des catégories fondamentales de l'entendement humain, en dehors du contexte socio-politique. Les discours sont-ils totalement inintentionnels? N'y a-t-il rien en dehors des structures du langage? A force d'autonomiser la sphère du sacré pour éviter tout réductionnisme arbitraire, on aboutit en vérité à des systèmes de représentations riches de sens mais dont on se demande à quoi ils servent, comment ils sont nés et quel est leur devenir. Doit-on admettre qu'un paysage urbain ne renvoie à rien d'autre qu'à d'autres textes par un jeu infini de citations, sans jamais acquérir de réalité historique? C'est difficilement recevable. Les villes ne sont pas, après tout, sorties comme par enchantement des structures de l'esprit humain. Comme les textes, elles méritent d'être mises en situation. Discours et pratiques s'y mêlent toujours de manière inextricable; le fait politique y est partout présent, y compris dans les civilisations de type traditionnel où il reste souvent inclus dans son expression symbolique, comme englobé par la religion.

Nous voici revenus au point de départ. Duncan a tenté d'appréhender conjointement deux modalités différentes de la ville: d'un côté sa symbolique et son langage, de l'autre ses aspects politiques. Faute d'avoir posé au départ les bonnes questions, il ne réussit cependant qu'à moitié dans son entreprise. Plutôt que d'éliminer les défauts inhérents à chaque approche prise isolément, il ne fait au bout du compte que les cumuler : idéaliste quand il parle de symbole, il s'avère fonctionnaliste quand il traite de politique. Les écueils étaient, il est vrai, difficiles à éviter tant les deux démarches correspondent à des champs disciplinaires tenus pour distincts. Les anthropologues se préoccupent surtout des structures de signification, ils cherchent à saisir le fonctionnement d'une société à travers ses rituels. Les historiens, pour leur part, placent les structures de pouvoir au cœur de leur analyse et s'intéressent davantage à l'action des cérémonies sur les sociétés ${ }^{23}$. Entre les deux approches les points de rencontre ne sont guère nombreux et toujours conflictuels. Il convient aujourd'hui de sortir de cette fausse alternative et de considérer les vues en présence non pas comme concurrentes, exclusives l'une de l'autre, mais concourantes. Une ville, un monument sont à la fois un événement et une structure. Un rituel, un symbole, ont toujours deux aspects : l'un instrumental, efficient (à la fois symboliquement et socialement), l'autre plus sémantique, incorporé à un ensemble religieux. De telles corrélations constituent un des enjeux majeurs des études sociologiques et anthropologiques à venir; elles ouvrent un champ de réflexions critiques et imposent un effort de synthèse. 


\section{La ville: texte ou palimpseste?}

Cette perspective croisée devrait permettre de mieux saisir les phénomènes de rupture que Duncan, ici plus anthropologue qu'historien, n'a pas réellement situés ni expliqués. Rien ne nous dit en effet que les paysages architecturés de Kandy n'ont pas été resémantisés à certaines époques et chargés d'une signification nouvelle par rapport à celle du texte original. "Le plan urbain est une chose mouvante, un perpétuel compromis entre un passé qui cherche à se survivre et une volonté consciente qui croit pouvoir imposer sa loi à la vie ", écrivait déjà en 1952 Max Sorre, un des doyens de la géographie humaine en France, trop vite oublié ${ }^{24}$. Ne serait-il pas plus juste, pour intégrer cette dimension diachronique, de parler de palimpseste et non de texte? Toutes les villes asiatiques ont vu des héritages culturels différents se fondre ou s'opposer à l'intérieur de leurs enceintes. Ceylan n'est pas la seule zone d'Asie du Sud où s'est établie une combinaison durable entre hindouisme et bouddhisme; la vallée de Kathmandou présente une symbiose similaire, parfaitement réussie en dépit des multiples tensions qui persistent entre les prêtres des deux confessions. Dans un cas comme dans l'autre, ces deux grandes religions écrites n'ont pas réussi, au demeurant, à gommer les vieilles pratiques ou croyances préexistantes, lesquelles, bien que greffées à de savantes théologies, continuent d'être inscrites sur le sol à l'état de traces. Les espaces urbains de cette partie du monde ont donc été continuellement raturés, effacés, réécrits au fil des années, recomposant à chaque époque un texte inédit à partir d'éléments anciens et nouveaux. Il serait fâcheux que l'anthropologue de la ville ne tienne pas compte de ces changements et ne contextualise pas davantage la structure symbolique dont on a montré plus haut toute l'importance dans les cités pré-industrielles. L'analyse des faits religieux s'est trop longtemps cantonnée dans l'intemporel. Elle a trop souvent entretenu le projet grandiose mais peu réaliste de construire une science unifiée de toute chose qui dévoilerait les structures logiques du monde grâce à l'étude des représentations. Même s'il relève de mécanismes symboliques très profondément enfouis dans la mémoire de l'humanité, et par conséquent relativement stables, le message que nous transmettent les cités n'est jamais indépendant des événements historiques. Il est constamment redéfini, actualisé par les péripéties de l'actualité et ne devient intelligible qu'au regard des perturbations du moment. En un mot, ce sont les espaces urbains eux-mêmes qui imposent une collaboration aux anthropologues et aux historiens.

Gérard ToFrin

CNRS, Paris

\section{NOTES}

1. F. Снолу, «Sémiologie et urbanisme», dans Le sens de la ville, Paris, Éditions du Seuil, 1972, pp. 11-30.

2. R. BARTHES, «Sémiologie et urbanisme», L'Architecture aujourd'hui, $1970, \mathrm{n}^{\circ}$ CLIII, pp. 11-13, et L'empire des signes, Paris, Skira, 1970.

3. T. PAvEL, Le mirage linguistique. Essai sur la modernisation intellectuelle, Paris, Les Éditions de Minuit, 1988.

4. N'étant pas spécialiste de Ceylan, je laisse à d'autres le soin d'apprécier l'exactitude du tableau présenté. Mon commentaire portera davantage sur l'apport de ce livre à l'étude du fait urbain en Asie du Sud et sur ses ambitions théoriques. 


\section{TERRITOIRES URBAINS}

5. G. Obeyesekere, "The great tradition and the little in the perspective of Sinhalese Buddhism ", Journal of Asian Studies, 1963, vol. 22, pp. 139-153, et The cult of the Godess Pattini, Chicago, Univ. of Chicago Press, 1984.

6. R. P. Kangle, The Kautilîya Arthaçâstra, 2.4.1. (1972, Bombay).

7. P. Wheatley, The Pivot of the Four Quarters, Chicago, Aldine, 1971.

8. G. Toffin, «La notion de ville dans une société asiatique traditionnelle: l'exemple des Néwar de la vallée de Kathmandou », L'Homme, 1982, XXII, 4, pp. 101-113. 1978.

9. H. L. Seneviratne, Rituals of the Kandyan State, Cambridge, Cambridge Univ. Press,

10. D. Winslow, «A political geography of deities: space and the pantheon in Sinhalese Buddhism », Journal of Asian Studies, 1984, vol. 43, pp. 273-291.

11. A. Cauquelin, L'invention du paysage, Paris, Plon, 1989, p. 144.

12. Par exemple V. Barré, P. Berger, L. Fevelle et G. Toffin, Panauti, une ville au Népal, Paris, Berger-Levrault, 1981.

13. Il convient de préciser que ces préceptes varient selon les textes (Mayamata, Arthâçastra, etc.) et la catégorie du roi.

14. Cf. V. BARRÉ et al., op. cit.

15. M. Roncayolo, La ville et ses territoires, Paris, Gallimard, «Folio», 1990, p. 90.

16. G. Bataille, CEuvres complètes, t. XII, Paris, Gallimard, 1980, p. 121.

17. P. VEYNE, "Conduites sans croyances et œuvres d'art sans spectateurs », Diogène, n 143, 1988, p. 11.

18. Idem, p. 11.

19. F. StaAl, «The Sound of Religion", Numen, vol. XXXIII, 1986, p. 204.

20. C. GeERTz, Negara. The theatre state in nineteenth century Bali, Princeton, Princeton Univ. Press, 1980.

21. J. BAzIN, "Donner à voir et faire entendre: symboliques de l'apparence», Annuaire. Comptes rendus des cours et conférences en 1988-1989, Paris, EHESS, 1990, pp. 268-269.

22. Idem, p. 269.

23. Cf. par exemple David Cannadine, «Introduction : Divine rites of kings », dans Rituals of royalty. Power and ceremonial in traditional societies, D. CANNADINE et S. PRICE éds, Cambridge Univ. Press, 1987, pp. 1-19.

24. Max Sorre, Les Fondements de la géographie humaine, Paris, Armand Colin, t. II, 1952, p. 260. 